\title{
American College of Sports Medicine
}

\author{
Posicionamento Oficial
}

\section{A quantidade e o tipo recomendados de exercícios para o desenvolvimento e a manutenção da aptidão cardiorrespiratória e muscular em adultos saudáveis}

\section{RESUMO}

Foi demonstrada a eficiência do exercício regular com uma determinada combinação de freqüência, intensidade e duração para a obtenção de um efeito de treinamento. A interação desses fatores fornece o estímulo de sobrecarga. Em geral, quanto mais baixo o estímulo, menores são os efeitos de treinamento e quanto maior o estímulo, maior é o efeito. Em conseqüência da especificidade do treinamento e da necessidade de manter a força e endurance dos principais grupos musculares e a flexibilidade das principais articulações, recomendamos um programa completo que inclua exercícios contra resistência e de flexibilidade. Embora a idade por si só não seja fator limitante para o treinamento, é prudente adotar uma abordagem mais gradual na prescrição para indivíduos mais idosos. Também foi demonstrado que o treinamento de endurance com frequiência inferior a duas vezes por semana, a intensidade inferior a $50 \%$ do consumo máximo de oxigênio e com duração inferior a dez minutos por dia é insuficiente para o desenvolvimento e a manutenção de aptidão física em adultos saudáveis.

$\mathrm{Na}$ interpretação deste posicionamento oficial, deve-se reconhecer que as recomendações devem ser utilizadas no contexto das necessidades, dos objetivos e da capacidade inicial dos participantes. Levando isso em conta, deve-se planejar uma programação distribuindo a quantidade total do tempo e da intensidade do esforço para os diferentes componentes do programa de treinamento - endurance cardiorrespiratória, força e endurance musculares. Recomenda-se também um aquecimento e volta à calma adequados que incluam exercícios de flexibilidade. O importante fator é planejar um programa para o indivíduo para fornecer a quantidade adequada de atividade física para atingir um benefício máximo com

Este posicionamento oficial substitui o de 1978 "A quantidade e o tipo recomendados de exercício para o desenvolvimento e a manutenção da aptidão física em adultos saudáveis". um risco mínimo. Deve-se dar ênfase aos fatores que resultem em uma modificação permanente do estilo de vida e estimulem a atividade física para o resto da vida.

Cada vez mais indivíduos estão se envolvendo em treinamento de endurance e outras formas de atividade física, e dessa forma torna-se clara a necessidade de estabelecer orientações para a prescrição de exercícios. Com base nas evidências científicas existentes a respeito de prescrição de exercícios para adultos saudáveis e na necessidade de orientações, o Colégio Americano de Medicina do Esporte estabelece as seguintes recomendações para a quantidade e o tipo de treinamento necessários para o desenvolvimento e a manutenção da aptidão cardiorrespiratória, composição corporal e força e endurance musculares no adulto saudável:

1. Freqüência de treinamento: 3 a 5 vezes por semana.

2. Intensidade de treinamento: 60 a $90 \%$ da freqüência cardíaca máxima $\left(\mathrm{FC}_{\text {máx }}\right)$, ou 50 a $85 \%$ do consumo máximo de oxigênio $\left(\mathrm{VO}_{2 \operatorname{máx}}\right)$ ou da reserva de $\mathrm{FC}_{\text {máx }}$ *.

3. Duração do treinamento: 20 a 60 minutos de atividade aeróbica contínua. A duração está relacionada com a intensidade da atividade; dessa forma, uma intensidade mais baixa deve ser mantida por um período maior de tempo. Pela importância da "aptidão total" e o fato de ser mais prontamente atingida em programas de duração mais longa, e devido aos riscos potenciais e problemas de aderência associados com atividades de alta intensidade, recomenda-se atividade de intensidade leve a moderada com maior duração para o adulto não atleta.

4. Tipo de atividade: qualquer atividade que mobilize grandes grupos musculares, possa ser mantida continuamente e seja de natureza rítmica e aeróbica, como, por exemplo, caminhada, corrida/jogging, andar de bicicleta ou exercício no

\footnotetext{
* A reserva de $\mathrm{FC}_{\text {máx }}$ é calculada a partir da diferença entre a $\mathrm{FC}$ máxima e de repouso. Para calcular a intensidade do treinamento, um percentual desse valor é adicionado à FC de repouso e expresso como um percentual da reserva de $\mathrm{FC}_{\text {máx }}{ }^{62}$.
} 
cicloergômetro, esqui cross-country (de planície), dança, pular corda, remo, subir escadas, nadar, patinar e diversas outras atividades lúdicas de endurance.

5. Treinamento contra resistência: o treinamento de força de intensidade moderada, suficiente para desenvolver e manter a massa corporal magra (MCM) deve fazer parte de um programa de aptidão física de um adulto. Uma série de 8 a 12 repetições de oito a dez exercícios que condicionem os principais grupos musculares pelo menos duas vezes por semana são o mínimo recomendado.

\section{FUNDAMENTOS CIENTÍFICOS}

\section{Introdução}

Freqüentemente são feitas as perguntas: "Quanto exercício é suficiente?" e "Qual tipo de exercício é o melhor para desenvolver e manter a aptidão física?" Sabe-se que o termo "aptidão física" é composto por uma série de características incluídas nas amplas categorias aptidão cardiorrespiratória, composição corporal, força e endurance musculares e flexibilidade. Neste contexto a aptidão é definida como a capacidade de desempenhar níveis moderados a intensos de atividade física sem fadiga exagerada e a possibilidade de manter esta capacidade ao longo da vida ${ }^{1}$. Também se reconhece que a resposta adaptativa ao treinamento é complexa e inclui fatores periféricos, centrais, estruturais e funcionais ${ }^{2,3}$. Embora muitas dessas variáveis e suas respostas adaptativas ao treinamento tenham sido documentadas, a falta de dados mais detalhados e comparativos em relação à freqüência, intensidade e duração do treinamento faz com que sejam inadequados para uso como modelos comparativos. Dessa forma, em relação às questões acima, a aptidão é limitada principalmente às alterações do $\mathrm{VO}_{2 \text { máx }}$, força e endurance musculares e composição corporal, que inclui a massa corporal total, a massa de gordura (MG) e a MCM. Ainda, a fundamentação científica utilizada para este posicionamento oficial será dividida em programas para aptidão cardiorrespiratória e controle ponderal e programas para força e endurance musculares.

Aptidão versus benefícios de saúde do exercício. Desde que o posicionamento oficial original foi publicado em 1978, uma importante distinção foi feita entre a atividade física relacionada com saúde ou aptidão física. Foi colocado que a quantidade e o tipo de exercícios necessários para atingir benefícios relacionados com a saúde podem ser diferentes daqueles recomendados para melhorar a aptidão física. Está claro que níveis menores de atividade física do que os recomendados por este posicionamento oficial reduzem o risco para o desenvolvimento de algumas doenças crônico-degenerativas e ainda podem não ser o suficiente para aumentar o $\mathrm{VO}_{2 \max }{ }^{1,4-6}$. O ACSM reconhece os potenciais benefícios para a saúde proporcionados pelo exercício regular realizado mais freqüentemente e por uma maior duração, mas em intensida- des menores do que as prescritas neste posicionamento oficial $^{4,7-10}$. O ACSM abordará a quantidade adequada de atividade física necessária para produzir benefícios em termos de saúde em um outro posicionamento.

Necessidade de padronizar os procedimentos e relatos de resultados. Apesar de uma grande quantidade de informações disponíveis sobre o treinamento do organismo humano, a falta de padronização dos protocolos e procedimentos de testes, da metodologia em relação aos procedimentos de treinamentos e o desenho do experimento, e de uma precisão da documentação e no relato da quantidade e da quantidade de treinamento prescrito fazem com que a interpretação seja difícil ${ }^{1,11-14}$. A interpretação e a comparação dos resultados também são dependentes do nível inicial de aptidão ${ }^{15-21}$, duração do período de treinamento do estudo ${ }^{13,22-27} \mathrm{e}$ da especificidade do teste e do treinamento ${ }^{2,3,13,16,28,29}$. Por exemplo, os dados de estudos envolvendo treinamento utilizando indivíduos com diferentes níveis de $\mathrm{VO}_{2 \max }$, massa corporal total e MG mostraram mudanças em relação aos seus valores iniciais ${ }^{19,20,30-34}$; ou seja, quanto menor o $\mathrm{VO}_{2 \text { máx }}$ inicial maior o percentual de melhora encontrado e quanto maior a MG, maior a redução. Ainda, dados avaliando a treinabilidade com a idade, comparações das diferentes magnitudes e quantidades de esforço e comparações da treinabilidade de homens e mulheres podem ter sido influenciados pelos níveis de aptidão iniciais.

Levando em conta o fato de que as melhoras das variáveis discutidas neste posicionamento continuam por meses de treinamento ${ }^{13,26,27,35,36}$, é razoável acreditar que estudos conduzidos por algumas poucas semanas possuem certas limitações. Indivíduos sedentários de meia-idade e idosos podem precisar de várias semanas para se adaptarem às fases iniciais do treinamento e dessa forma necessitam de um período de adaptação maior para obter o máximo benefício de um programa. Por exemplo, Seals et al. ${ }^{27}$ submeteram indivíduos de 60 a 69 anos a um período de treinamento de 12 meses. Esses indivíduos tiveram uma melhora de $12 \%$ no $\mathrm{VO}_{2 \text { max }}$ após seis meses de caminhada em intensidade moderada. Um aumento adicional de $18 \%$ ocorreu nos seis meses seguintes, com a introdução do jogging. A duração ideal de um estudo é difícil de determinar, mas um período 15 a 20 semanas pode ser um bom padrão mínimo. Embora seja difícil controlar os estudos envolvendo treinamento físico por mais de um ano, há necessidade de estudar esse efeito. Conforme estabelecido antes, doses menores de exercício podem melhorar o $\mathrm{VO}_{2 \max }$ e controlar ou manter a composição corporal, mas de forma mais lenta.

Embora a maior parte das informações a respeito do treinamento descritas neste posicionamento tenham sido realizados com homens, as evidências disponíveis indicam que as mulheres tendem a se adaptar ao treinamento de endurance da mesma forma que os homens ${ }^{37-48}$. 


\section{Prescrição de exercícios para aptidão cardiorrespirató- ria e controle ponderal}

A prescrição de exercícios tem como fundamentos a freqüência, intensidade, duração e o tipo de atividade (de natureza aeróbica, p.ex., listados no número 4 acima), além do nível inicial de aptidão. As seguintes observações avaliam estes fatores acima e se baseiam em estudos envolvendo programas de treinamento de endurance com duração de 6 a 12 meses.

Aumentos do $\mathrm{VO}_{2 \operatorname{máx}}$ estão diretamente relacionados com a freqüência ${ }^{14,24,49-57}$, intensidade ${ }^{14,17,49,50,52-54,56,58-64} \mathrm{e}$ duração ${ }^{14}$, $32,49,52-54,56,59,60,64-69$ do treinamento. Na dependência da quantidade e do tipo de treinamento, os aumentos do $\mathrm{VO}_{2 \max }$ variam entre 5 e $30 \%{ }^{11,13,14,27,43,52-54,56,59,60,67,69-90}$. Estes estudos mostram que um aumento do $\mathrm{VO}_{2 \text { máx }}$ de no mínimo $15 \%$ é geralmente atingido em programas que seguem as orientações estabelecidas mais acima. Embora alterações do $\mathrm{VO}_{2 \text { máx }}$ maiores do que $30 \%$ tenham sido demonstradas, estas geralmente estão associadas com grandes reduções da massa corporal total e da MG, em pacientes cardiopatas ou em indivíduos com um nível inicial de aptidão muito baixo. Ainda, como resultado de fadiga dos membros inferiores ou falta de motivação, indivíduos com uma aptidão física inicial baixa podem ter valores de $\mathrm{VO}_{2 \text { máx }}$ espuriamente baixos. Klissouras $^{91}$ e Bouchard ${ }^{92}$ mostraram que a variação humana do $\mathrm{VO}_{2 \text { máx }}$ com o treinamento é importante e relacionada com o nível fenotípico atual. Isto é, há um estado pré-treinamento determinado geneticamente e uma capacidade de adaptação com o treinamento. Dessa forma, os resultados fisiológicos devem ser interpretados de acordo com a variação genética e a qualidade e quantidade de treinamento realizado.

Intensidade-duração. A intensidade e duração do treinamento estão inter-relacionadas, sendo a quantidade total de trabalho realizado um importante fator para a melhora de aptidão física ${ }^{11,14,20,25,35,45,46,72,84,87,93-95}$. Embora investigações mais esclarecedoras sejam necessárias, as evidências atuais sugerem que quando o exercício é realizado acima de um limiar mínimo de intensidade, a quantidade total de trabalho realizado é um importante fator no desenvolvimento ${ }^{20,35,37,55}$, ${ }^{84,87}$ e na manutenção ${ }^{96}$ da aptidão. Isto é, o aumento será semelhante para atividades realizadas com uma baixa intensidade-longa duração comparando-se com alta intensidadecurta duração se os gastos energéticos totais destas atividades forem semelhantes. Um exercício de alta intensidade está associado com um maior risco cardiovascular ${ }^{97}$, maior risco de lesões ortopédicas ${ }^{13,98}$ e menor aderência ao treinamento do que o exercício de baixa intensidade ${ }^{98-101}$. Portanto, os programas que enfatizam o treinamento de intensidade baixa a moderada com maior duração são recomendados para a maior parte dos adultos.

O limiar de intensidade mínima de treinamento para melhorar o $\mathrm{VO}_{2 \text { máx }}$ é de aproximadamente $60 \%$ da $\mathrm{FC}_{\text {máx }}(50 \%$ do $\mathrm{VO}_{2 \text { máx }}$ ou da reserva de $\left.\mathrm{FC}_{\text {máx }}\right)^{61,62}$. Estes $50 \%$ da reserva de $\mathrm{FC}_{\text {máx }}$ representam uma frequiência cardíaca $(\mathrm{FC})$ de aproximadamente 130 a $135 \mathrm{bpm}$ para indivíduos jovens. Devido à relação da $\mathrm{FC}_{\text {máx }}$ com a idade, a $\mathrm{FC}$ absoluta para atingir este limiar está inversamente relacionada com a idade, e fica na faixa entre 105 e $115 \mathrm{bpm}$ para pessoas mais idosas ${ }^{27,43,102}$. Os pacientes que tomam betabloqueadores podem ter valores de FC significativamente mais baixos ${ }^{103}$. O nível inicial de aptidão é outra importante consideração na prescrição de exercícios ${ }^{19,20,45,58,104}$. O indivíduo com um nível baixo de aptidão pode atingir um efeito significativo de treinamento com uma FC de treinamento na faixa de 40 a $50 \%$ da reserva de $\mathrm{FC}_{\text {máx }}$, enquanto que indivíduos com níveis mais altos de aptidão física necessitam um estímulo maior ${ }^{14,17,56,102}$.

Classificação da intensidade do exercício. A classificação da intensidade do exercício e a sua padronização para a prescrição de exercícios baseada em uma sessão de treinamento com duração de 20 a 60 minutos têm sido confusas, mal interpretadas e freqüentemente fora de contexto. O sistema de classificação mais reproduzido baseia-se no gasto energético $\left(\mathrm{kcal} \cdot \mathrm{min}^{-1} \cdot \mathrm{kg}^{-1}\right)$ de tarefas industriais ${ }^{105,106}$. Os dados originais para este sistema de classificação foram publicados por Christensen ${ }^{107}$ em 1953 e foram baseados no gasto energético de trabalhadores de indústria siderúrgica por oito horas diárias. A classificação de tarefas industriais e de lazer utilizando valores absolutos para gasto energético foram importantes para uso nas áreas ocupacional e nutricional. Embora esta classificação tenha ampla aplicação na medicina e, em particular, para fazer recomendações para controle de peso e determinação de tarefas no trabalho, possui pouco ou nenhum valor para programas de exercício preventivos e de reabilitação. Extrapolar valores absolutos de gasto energético para uma tarefa industrial baseada em uma jornada de trabalho de oito horas para um regime de treinamento físico de 20 a 60 minutos de duração não faz nenhum sentido. Por exemplo, caminhada e corrida/jogging podem ser feitos em uma ampla variedade de velocidades; dessa forma, a intensidade relativa se torna importante sob estas condições. Pelo fato de que os regimes de treinamento de endurance recomendados pelo ACSM para adultos não atletas são planejados para 60 minutos ou menos de atividade física, recomendamos o sistema de classificação de intensidade de treinamento mostrado na tabela $1^{13}$. O uso de uma duração adequada para o treinamento e de uma intensidade relativa de esforço individualizada torna este sistema aplicável tanto a jovens quanto a indivíduos de meia-idade e idosos, bem como pacientes com capacidade funcional limitada ${ }^{13,49,108}$.

A tabela 1 também descreve a relação entre a intensidade relativa de esforço, baseada no percentual da $\mathrm{FC}_{\text {máx }}$, no percentual da reserva de $\mathrm{FC}_{\text {máx }}$, ou no percentual do $\mathrm{VO}_{2 \text { máx }}$, e o índice de percepção de esforço (IPE) da escala de Borg ${ }^{108-110}$. $\mathrm{O}$ uso da FC como uma estimativa da intensidade do treinamento é o padrão comum ${ }^{13,49}$. 
TABELA 1

Classificação da intensidade do exercício baseada em um treinamento de endurance de 20 a 60 minutos

\begin{tabular}{lccl} 
Intensidade relativa (\%) & $\begin{array}{c}\text { Índice de } \\
\text { percepção do } \\
\text { esforço }\end{array}$ & $\begin{array}{l}\text { Classificação } \\
\text { de intensidade }\end{array}$ \\
\hline FC $_{\text {máx }} *$ & $\begin{array}{c}\text { VO }_{\text {2máx }} \text { *u ou } \\
\text { reserva } \\
\text { da FC }\end{array}$ & & \\
$<35 \%$ & $<30 \%$ & $<10$ & Muito leve \\
$35-59 \%$ & $30-49 \%$ & $10-11$ & Leve \\
$60-79 \%$ & $50-74 \%$ & $12-13$ & $\begin{array}{l}\text { Moderada } \\
\text { (algo pesada) }\end{array}$ \\
$80-89 \%$ & $75-84 \%$ & $14-16$ & $\begin{array}{l}\text { Pesada } \\
\text { Muito pesada }\end{array}$ \\
\hline
\end{tabular}

Tabela extraída de: Pollock ML, Wilmore J H. Exercise in health and disease: evaluation and prescription for prevention and rehabilitation. $2^{\text {nd }}$ ed. Philadelphia: WB Saunders, 1990. Publicada com permissão.

* $\mathrm{FC}_{\text {máx }}=$ freqüência cardíaca máxima; $\mathrm{VO}_{2 \text { máx }}=$ consumo máximo de oxigênio.

O uso da escala de Borg tornou-se um instrumento válido na monitorização da intensidade em programas de treinamento físico ${ }^{13,108,111,112}$. Esta escala é geralmente considerada um auxiliar da FC na monitorização da intensidade do esforço, mas desde que a relação entre a FC e a escala de Borg para um determinado indivíduo seja conhecida, o IPE pode ser utilizado no lugar da $\mathrm{FC}^{13,113}$. Não seria o caso de determinados tipos de pacientes, nos quais um controle mais preciso da FC pode ser fundamental para a segurança do procedimento.

Freqüiência. A quantidade de melhora do $\mathrm{VO}_{2 \text { máx }}$ tende a um platô quando a freqüência de treinamento aumenta além de três vezes por semana ${ }^{11,13,51}$. O valor da melhora adicional do $\mathrm{VO}_{2 \text { máx }}$ com treinamento acima de cinco vezes por semana é de pequeno a inaparente ${ }^{11,52-54,114}$. Treinar menos do que duas vezes por semana não leva a nenhuma alteração notável do $\mathrm{VO}_{2 \text { máx }} 11,14,51,56,59,64$.

Tipo. Se a frequiência, a intensidade e a duração do treinamento são semelhantes (gasto calórico total), as adaptações ao treinamento parecem ser independentes do tipo de atividade aeróbica ${ }^{28,64,115}$. Portanto, uma variedade de atividades de endurance, como, por exemplo, as listadas no início deste documento, podem ser utilizadas para obter o mesmo efeito de treinamento.

Atividades de endurance que envolvam corrida e saltos são consideradas de alto impacto e geralmente produzem lesões bem mais importantes para indivíduos iniciantes e mesmo experientes do que aquelas de baixo impacto e exercícios nos quais não se sustenta o próprio peso ${ }^{63,84,98,116-120}$. Isso é particularmente claro no idoso ${ }^{13}$. Corredores iniciantes tiveram aumento de lesões nos pés, pernas e joelhos quando o treinamento foi realizado mais do que três vezes por semana e com duração acima de 30 minutos por sessão ${ }^{118}$. Treina- mento intervalado de alta intensidade (corrida-caminhada) comparado com um jogging contínuo também estava associado com maior incidência de lesões ${ }^{95,98}$. Dessa forma, devese tomar cuidado quando se recomenda o tipo de atividade e na prescrição de exercícios para o iniciante. Lesões ortopédicas relacionadas a excesso de sobrecarga aumentam linearmente em corredores quando estes realizam essas ativida$\operatorname{des}^{116,119}$. Assim, há necessidade de mais pesquisas sobre os efeitos de diferentes tipos de atividades e a quantidade e qualidade do treinamento sobre as lesões, tanto a curto quanto a longo prazo.

Uma atividade como treinamento de peso não deve ser considerada como meio de treinamento para aumentar o $\mathrm{VO}_{2 \text { máx }}$, mas tem importante valor no aumento da força e endurance musculares e MCM ${ }^{121-125}$. Estudos que avaliaram treinamento de peso em circuito (treinamento de peso conduzido de forma praticamente contínua, com pesos moderados, utilizando 10 a 15 repetições por estação de exercício, com intervalos de 15 a 30 segundos entre elas) mostraram aumento médio do $\mathrm{VO}_{2 \text { máx }}$ de $6 \%^{122,126-133}$. Dessa forma, o treinamento de peso em circuito não é recomendado como a única atividade utilizada em programas de exercício para melhorar o $\mathrm{VO}_{2 \text { máx }}$.

Idade. A idade em si não parece ser um obstáculo para o treinamento de endurance. Embora alguns estudos mais antigos tenham mostrado um menor efeito do treinamento em indivíduos de meia-idade e idosos ${ }^{69,134-137}$, estudos mais recentes mostram que os aumentos relativos do $\mathrm{VO}_{2 \text { máx }}$ são semelhantes aos obtidos em indivíduos mais jovens $27,43,70,138-$ 141. Embora mais estudos sejam necessários sobre a taxa de aumento do $\mathrm{VO}_{2 \text { máx }}$ com o treinamento em diferentes idades, hoje parece que os indivíduos mais idosos necessitam períodos mais longos de tempo para se adaptarem ${ }^{27,135,139}$. Estudos mais antigos que mostraram pouco ou nenhum aumento do $\mathrm{VO}_{2 \text { máx }}$ tiveram curta duração ${ }^{134}$ ou o exercício foi realizado em uma intensidade moderada a baixa ${ }^{135}$, dificultando dessa forma a interpretação dos resultados.

Embora o $\mathrm{VO}_{2 \text { máx }}$ diminua e a massa corporal total e a $\mathrm{MG}$ aumentem com a idade, evidências sugerem que essa tendência pode ser alterada com treinamento de endurance ${ }^{13,35}$, 36,142-144. Uma redução de $9 \%$ do $\mathrm{VO}_{2 \text { máx }}$ por década para adultos sedentários após os 25 anos de idade foi demonstrada ${ }^{145,146}$, mas para indivíduos ativos esta redução pode ser inferior a $5 \%$ por década ${ }^{145-148}$. Estudos que acompanharam indivíduos durante dez ou mais anos mostraram que aqueles que continuavam treinando em nível semelhante mantinham sua aptidão cardiorrespiratória ${ }^{143,144,149,150}$. Um estudo com corredores de competição idosos mostrou valores progressivamente menores de $\mathrm{VO}_{2 \text { máx }}$ da quarta à sétima décadas de vida, mas também mostraram menor volume de treinamento nos grupos de maior idade ${ }^{151}$. Dados de acompanhamento de dez anos desses mesmos atletas (50 a 82 anos de idade) mostraram que o $\mathrm{VO}_{2 \text { máx }}$ mantinha-se inalterado quando a quantida- 
de e a qualidade do treinamento mantinham-se a mesma ${ }^{150}$. Assim, o estilo de vida desempenha importante papel na manutenção da aptidão. São necessários mais estudos em relação ao treinamento a longo prazo (quantidade e qualidade), tanto para competidores, quanto para não-competidores, e à função fisiológica com o aumento da idade, antes que afirmações mais definitivas sejam feitas.

Manutenção do efeito de treinamento. Para manter o efeito de treinamento, o exercício deve ser realizado regularmente ${ }^{40,81,152-157}$. Uma redução importante da aptidão cardiorrespiratória ocorre após duas semanas de destreinamento ${ }^{153,156}$, com os participantes chegando próximo aos níveis pré-treinamento após dez semanas ${ }^{40}$ a oito meses sem treinamento ${ }^{155}$. Uma perda de $50 \%$ da melhora inicial do $\mathrm{VO}_{2 \operatorname{máx}}$ foi mostrada após 4 a 12 semanas sem treinamento ${ }^{40,156,158}$. Os indivíduos que foram submetidos a anos de treinamento contínuo mantêm alguns benefícios por períodos mais prolongados sem treinamento do que indivíduos que estão em treinamento há menos tempo ${ }^{153}$. Enquanto que uma parada total no treinamento leva a importantes reduções do $\mathrm{VO}_{2 \text { máx }}$, uma redução da carga de treinamento mostra pequena ou nenhuma redução durante períodos de 5 a 15 semanas ${ }^{52-54,152,156}$. Hickson et al., em uma série de experimentos em que a freqüência ${ }^{52}$, a duração $0^{53}$ ou a intensidade ${ }^{54}$ do treinamento foram manipuladas, observaram que se a intensidade do treinamento permanecia inalterada, o $\mathrm{VO}_{2 \text { máx }}$ mantinha-se por até 15 semanas com reduções da freqüência e duração do treinamento de até dois terços. Quando a freqüência e a duração do treinamento permaneciam constantes e a intensidade do treinamento era reduzida em um terço ou dois terços, o $\mathrm{VO}_{2 \text { máx }}$ diminuía significativamente. Achados semelhantes foram observados em relação ao treinamento de força. Quando o treinamento de força era reduzido de três ou duas vezes por semana para pelo menos uma vez por semana, a força se mantinha por 12 semanas de treinamento reduzido ${ }^{42}$. Assim, parece que perder uma sessão de exercício de vez em quando ou reduzir o treinamento por até 15 semanas não afetará de maneira adversa o $\mathrm{VO}_{2 \text { máx }}$ ou a força ou endurance musculares, desde que a intensidade do treinamento seja mantida.

Embora muitos estudos recentes tenham fornecido novos dados em relação à quantidade adequada de exercício, são necessários estudos para avaliar a taxa de aumento ou redução da aptidão quando se variam as cargas de treinamento e quando se reduz o treinamento, conforme o nível de aptidão, idade e tempo prévio de treinamento. Ainda, são necessárias mais informações para identificar melhor o mínimo nível de exercício necessário para manter a aptidão.

Controle ponderal e composição corporal. Embora haja uma variabilidade na resposta ao exercício para as modificações da composição corporal, a massa corporal total e a MG geralmente se reduzem com programas de treinamento de endurance ${ }^{12,13,159}$, enquanto que a MCM permanece constan- te $\mathrm{e}^{11,12,13,160}$ ou aumenta discretamente ${ }^{161,162}$. Por exemplo, Wilmore ${ }^{159}$ relatou os resultados de 32 estudos que preencheram os critérios para melhorar a aptidão cardiorrespiratória que são enfatizados neste posicionamento oficial e encontrou uma redução média da massa corporal total de $1,5 \mathrm{~kg}$ e do percentual de gordura de $2,2 \%$. Os programas para redução de peso que utilizam uma manipulação dietética que produz diminuição mais acentuada da massa corporal total provocam uma redução tanto da MG como da $\mathrm{MCM}^{162-164}$. Quando estes programas são conduzidos junto com o treinamento físico, a redução da MCM é menor do que nos programas que utilizam somente dieta ${ }^{164,165}$. Os programas que são conduzidos com pelo menos três sessões semanais ${ }^{11,24,25,55,160}$, com uma duração de pelo menos 20 minutos $^{11,32,160}$ e com uma intensidade suficiente para provocar um gasto energético de aproximadamente 300kcal por sessão, são sugeridos como um nível limiar para a redução da massa corporal total e da $\mathrm{MG}^{11-13,35 \text {, }}$ 54,166. Demonstrou-se que um gasto de $200 \mathrm{kcal}$ por sessão também é útil para redução de peso se a frequiência for de pelo menos quatro vezes por semana ${ }^{167}$. Se o objetivo principal do programa de treinamento for a redução de peso, esquemas de maior frequiência semanal, maior duração da sessão e intensidade baixa a moderada são os ideais ${ }^{13,163}$. Programas com menos do que isso geralmente levam a uma pequena ou a nenhuma modificação da composição corporal ${ }^{11,12,63,68,160 \text {, }}$ 168-170. Aumentos importantes do $\mathrm{VO}_{2 \text { máx }}$ foram demonstrados com 10 a 15 minutos de treinamento de alta intensidade ${ }^{11,32}$, $50,56,64,88,136$; dessa forma, se a redução da massa corporal total e da MG não são importantes, pode-se recomendar esquemas de curta duração e alta intensidade para indivíduos saudáveis com baixo risco de doenças cardiovasculares e lesões ortopédicas.

\section{Prescrição de exercícios para força e "endurance" mus- culares}

A inserção deste assunto - treinamento contra resistência para desenvolver a força muscular - neste documento é justificada pela necessidade de um programa completo que exercite todos os principais grupos musculares. Dessa forma, a inclusão do treinamento contra resistência em programas de aptidão física para adultos é importante para o desenvolvimento e a manutenção da MCM. O efeito do treinamento físico é específico para o grupo muscular que é trabalha$\mathrm{do}^{2,3,16,29}$. Por exemplo, um treinamento dos membros inferiores não terá nenhum efeito nos músculos dos membros superiores e tronco. Um acompanhamento por dez anos de corredores da categoria master que continuaram o seu esquema de treinamento mas não faziam exercício para o tronco e membros superiores mostrou a manutenção do $\mathrm{VO}_{2 \text { máx }} \mathrm{e}$ uma redução de $2 \mathrm{~kg}$ na $\mathrm{MCM}^{150}$. A circunferência da perna se manteve, mas a circunferência do braço reduziu-se de forma importante. Esses dados indicam uma perda de massa muscular nas regiões não treinadas. Três dos atletas que pra- 
ticavam exercícios com pesos para os membros superiores e tronco mantiveram a sua MCM. Uma revisão realizada por Sale $^{29}$ documentou detalhadamente a especificidade do treinamento.

A especificidade do treinamento foi também abordada por Graves et $a l .{ }^{171}$. Utilizando um exercício de extensão bilateral das pernas, foram treinados quatro grupos: grupo A, com a primeira metade da amplitude do movimento; grupo $\mathrm{B}$, segunda metade da amplitude do movimento; grupo $\mathrm{AB}$, amplitude total do movimento; e um grupo-controle que não treinou. Os resultados mostraram claramente que o resultado do treinamento foi específico para a parte da amplitude de movimento treinada, sendo que o grupo $\mathrm{AB}$ atingiu os melhores resultados em toda a amplitude de movimento. Dessa forma, o treinamento contra resistência deve ser realizado em toda a amplitude de um determinado movimento, para que o benefício seja maior ${ }^{171,172}$.

A força e endurance musculares se desenvolvem pelo princípio da sobrecarga, ou seja, aumentando mais do que o habitual a resistência ao movimento, a frequiência ou a duração de uma determinada atividade $\mathrm{e}^{16,26,121,173,174}$. A força muscular se desenvolve mais facilmente quando se utilizam pesos maiores (que exijam uma tensão muscular máxima ou próxima à máxima) com poucas repetições, enquanto que a endurance muscular se desenvolve melhor com a utilização de pesos mais leves, com um grande número de repetições ${ }^{16,26 \text {, }}$ ${ }^{173,175}$. Até certo ponto, desenvolve-se tanto a força quanto a endurance muscular sob qualquer destas condições, mas cada sistema favorece mais o desenvolvimento de uma destas qualidades específicas ${ }^{16,26}$. Assim, para produzir aumentos tanto da força quanto da endurance musculares, os especialistas recomendam oito a 12 repetições por série.

Qualquer quantidade de sobrecarga resultará no aumento da força, mas um exercício em uma intensidade maior ou um

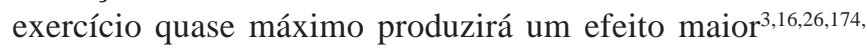
176,177. A intensidade do treinamento contra resistência pode ser manipulada variando o peso, o número de repetições, o tempo de intervalo entre as séries e o número de séries realizadas $^{16}$. Deve-se ter cuidado com treinamento de força que enfatiza contrações excêntricas, pois o potencial para lesões musculares é maior ${ }^{178,179}$.

A força e endurance musculares podem ser desenvolvidas através de exercícios estáticos e dinâmicos. Embora cada tipo de treinamento tenha os seus pontos fortes e fracos, recomenda-se exercícios dinâmicos para adultos saudáveis. O treinamento contra resistência para o participante mediano deve ser rítmico, em uma velocidade moderada a baixa, através de toda a amplitude do movimento e sem impedir a respiração normal. O treinamento contra resistência mais pesado pode provocar um importante aumento da pressão arterial sistólica e diastólica ${ }^{180,181}$.

A melhora esperada da força com o treinamento contra resistência é difícil de avaliar porque os aumentos da força são dependentes do nível inicial de força do participante,

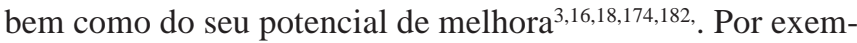
plo, Mueller e Rohmert ${ }^{18}$ encontraram aumentos da força de 2 a $9 \%$ por semana dependendo dos níveis iniciais. Embora a literatura mostre uma ampla faixa de melhora de força com os programas de treinamento contra resistência, a melhora média para homens e mulheres sedentários de meia-idade para até seis meses de treinamento é de 25 a 30\%. Fleck e Kraemer ${ }^{16}$, em uma revisão de 13 estudos representando diferentes tipos de treinamento dinâmico, mostraram uma melhora média da força no supino de $23,3 \%$ quando os indivíduos foram testados no equipamento no qual foram treinados e de $16,5 \%$ quando foram testados em ergômetros especiais isotônicos ou isocinéticos (seis estudos). Fleck e Kraemer $^{16}$ relataram também um aumento médio de $26,6 \%$ na força dos membros inferiores quando os indivíduos foram testados no equipamento no qual foram treinados e de $21,2 \%$ quando testados em equipamentos isotônicos e isocinéticos (cinco estudos). Os resultados de melhora da força resultantes de treinamento estático foram da mesma magnitude dos resultados com treinamento dinâmico ${ }^{22,42,116,171}$.

À luz da informações relatadas acima, estabelecemos as seguintes orientações para treinamento contra resistência para o adulto saudável médio. Pelo menos oito a dez exercícios envolvendo os principais grupos musculares devem ser realizados pelo menos duas vezes por semana. Pelo menos uma série de oito a 12 repetições que leve quase à fadiga deve ser realizada. Este padrão mínimo para treinamento contra resistência se baseia em dois fatores. Primeiro, o tempo que se leva para realizar um programa de exercícios completo é importante. Programas que tenham sessões com mais de 60 minutos de duração têm maiores taxas de abandono ${ }^{98}$. Segundo, embora uma maior freqüência de treinamento ${ }^{16,22,183}$ e séries adicionais ou combinações de séries e repetições tragam maiores ganhos de força ${ }^{3,16,26,121,174,175}$, a diferença é normalmente pequena. Por exemplo, Braith et al. ${ }^{22}$ compararam o treinamento duas vezes por semana com três vezes por semana por 18 semanas. Os indivíduos realizaram uma série de sete a dez repetições até a fadiga. O grupo de duas vezes teve um aumento de força de $21 \%$ e o grupo de três vezes teve um aumento de $28 \%$. Em outras palavras, com duas vezes semanais conseguiram-se $75 \%$ do que foi conseguido com três vezes por semana. Ainda, estes $21 \%$ conseguidos com duas vezes por semana representam 70 a $80 \%$ da melhora relatada por outros programas que utilizam uma maior freqüência semanal e combinações de séries e repetições ${ }^{16}$. Graves et al. ${ }^{42,171}$, Gettman et al. ${ }^{184}$, Hurley et al. ${ }^{129}$ e Braith et $a l{ }^{22}$ observaram que programas que utilizam uma série até a fadiga conseguem aumentos de força maiores do que $25 \%$. Embora os equipamentos utilizados para treinamento contra resistência possam proporcionar um estímulo mais graduado e quantitativo para sobrecarga do que os exercícios calistênicos tradicionais, estes e outros tipos de exercícios ainda 
podem ser utilizados de maneira eficiente para melhorar e manter a força muscular.

\section{REFERÊNCIAS}

1. Wilmore JH. Design issues and alternatives in assessing physical fitness among apparently healthy adults in a health examination survey of the general population. In: Drury TF, editor. Assessing physical fitness and activity in general population studies. Washington, DC: U.S. Public Health Service, National Center for Health Statistics, 1988 (in press).

2. Åstrand PO, Rodahl K. Textbook of work physiology. $3^{\text {rd }}$ ed. New York: McGraw-Hill, 1986:412-85.

3. Wilmore JH, Costill DL. Training for sport and activity. The physiological basis of the conditioning process. $3^{\text {rd }}$ ed. Dubuque, IA: Wm. C. Brown, 1988:113-212.

4. Haskell WL. Physical activity and health: need to define the required stimulus. Am J Cardiol 1985;55:4D-9D.

5. Haskel WH, Montoye HJ, Orenstein D. Physical activity and exercise to achieve health-related physical fitness components. Public Health Rep 1985;100:202-12.

6. Laporte RE, Adams LL, Savage DD, Brenes G, Dearwater S, Cook T. The spectrum of physical activity, cardiovascular disease and health: an epidemiologic perspective. Am J Epidemiol 1984;120:507-17.

7. Blair SN, Kohl III HW, Paffenbarger RS, Clark DG, Cooper KH, Gibbons LH. Physical fitness and all-cause mortality. A prospective study of healthy men and women. JAMA 1989;262:2395-2401.

8. Leon AS, Connett J, Jacobs DR, Rauramaa R. Leisure-time physical activity levels and risk of coronary heart disease and death: the multiple risk of coronary heart disease and death: the multiple risk factor intervention trial. JAMA 1987;258:2388-95.

9. Paffenbarger RS, Hyde RT, Wing AL, Hsieh C. Physical activity and allcause mortality, and longevity of college alumni. N Engl J Med 1986; 314:605-13.

10. Smith EL, Reddan W, Smith PE. Physical activity and calcium modalities for bone mineral increase in aged women. Med Sci Sports Exerc 1981;13:60-4.

11. Pollock ML. The quantification of endurance training programs. In: Wilmore JH, editor. Exercise and sport sciences reviews. New York: Academic Press, 1973:155-88.

12. Pollock ML, Jackson A. Body composition: measurement and changes resulting from physical training. Proceedings National College Physical Education Association for Men and Women, January, 1977:125-37.

13. Pollock ML, Wilmore JH. Exercise in health and disease: evaluation and prescription for prevention and rehabilitation. $2^{\text {nd }}$ ed. Philadelphia: W.B. Saunders, Co., 1990.

14. Wenger HA, Bell GJ. The interactions of intensity, frequency, and duration of exercise training in altering cardiorespiratory fitness. Sports Med 1986;3:346-56.

Traduzido por:

José Kawazoe Lazzoli

Professor da Universidade Federal Fluminense, Niterói, RJ

Membro das diretorias da Sociedade Brasileira de Medicina do Esporte e Sociedade de Medicina Desportiva do Rio de Janeiro

Diretor do Ergocenter - Instituto Petropolitano de Ergometria, Petrópolis, RJ

Traduzido com permissão por escrito do original The recommended quantity and quality of exercise for developing and maintaining cardiorespiratory and muscular fitness in healthy adults. Med Sci Sports Exerc 1990;22:265-74.
15. Ekblom B, Åstrand PO, Saltin B, Stenberg J, Wallstrom B. Effect of training on circulatory response to exercise. J Appl Physiol 1968;24: 518-28.

16. Fleck SJ, Kraemer WJ. Designing resistance training programs. Champaign, IL: Human Kinetics Books, 1987;15-46,161-2.

17. Gledhill N, Eynon RB. The intensity of training. In: Taylor AW, Howell ML, editors. Training scientific basis and application. Springfield, IL: Charles C. Thomas Co., 1972:97-102.

18. Mueller EA, Rohmert W. Die geschwindigkeit der muskelkraft zunahme bein isometrischen training. Int Z Angew Physiol 1963;19:403-19.

19. Saltin B, Hartley L, Kilbom A, Åstrand I. Physical training in sedentary middle-aged and older men. Scand J Clin Lab Invest 1969;24:323-34.

20. Sharkey BJ. Intensity and duration of training and the development of cardiorespiratory endurance. Med Sci Sports 1970;2:197-202.

21. Siegel W, Blomqvist G, Mitchell JH. Effects of a quantitated physical training program on middle-aged sedentary males. Circulation 1970; 41:19-29.

22. Braith RW, Graves JE, Pollock ML, Leggett SL, Carpenter DM, Colvin $\mathrm{AB}$. Comparison of two versus three days per week of variable resistance training during 10 and 18 week programs. Int J Sports Med 1989; 10:450-4.

23. Fox EL, Bartels RL, Billings CE, O'Brien R, Bason R, Mathews DK. Frequency and duration of interval training programs and changes in aerobic power. J Appl Physiol 1975;38:481-4.

24. Pollock ML, Cureton TK, Greninger L. Effects of frequency of training on working capacity, cardiovascular function, and body composition of adult men. Med Sci Sports 1969;1:70-4.

25. Pollock ML, Broida J, Kendrick Z, Miller HS, Janeway R, Linnerud AC. Effects of training two days per week at different intensities on middle-aged men. Med Sci Sports 1972;4:192-7.

26. Sale DG. Influence of exercise and training on motor unit activation. In: Pandolf KB, editor. Exercise and sport sciences reviews. New York: McMillan Publishing Co., 1987:95-152.

27. Seals DR, Hagberg JM, Hurley BF, Ehsani AA, Holloszy JO. Endurance training in older men and women. I. Cardiovascular responses to exercise. J Appl Physiol 1984;57:1024-9.

28. Pollock ML, Dimmick J, Miller HS, Kendrick Z, Linnerud AC. Effects of mode of training on cardiovascular function and body composition of middle-aged men. Med Sci Sports 1975;7:139-45.

29. Sale DG. Neural adaptation to resistance training. Med Sci Sports Exerc 1988;20:S135-45.

30. Boileau RA, Buskirk ER, Horstman DH, Mendez J, Nicholas W. Body composition changes in obese and lean men during physical conditioning. Med Sci Sports 1971;3:183-9.

31. Dempsey JA. Anthropometrical observations on obese and nonobese young men undergoing a program of vigorous physical exercise. Res Q 1964;35:275-87.

32. Milesis CA, Pollock ML, Bah MD, Ayres JJ, Ward A, Linnerud AC. Effects of different durations of training on cardiorespiratory function, body composition and serum lipids. Res Q 1976;47:716-25.

33. Moody DL, Kollias J, Buskirk ER. The effect of a moderate exercise program on body weight and skinfold thickness in overweight college women. Med Sci Sports 1969;1:75-80.

34. Moody DL, Wilmore JH, Girandola RN, Royce JP. The effects of a jogging program on the body composition of normal and obese high school girls. Med Sci Sports 1972;4:210-3.

35. Cureton TK. The physiological effects of exercise programs upon adults. Springfield, IL: Charles C. Thomas Co., 1969:3-6,33-77. 
36. Kasch FW, Phillips WH, Carter JEL, Boyer JL. Cardiovascular changes in middle-aged men during two years of training. J Appl Physiol 1972; 314:53-7.

37. Burke EJ. Physiological effects of similar training programs in males and females. Res Q 1977;48:510-7.

38. Drinkwater BL. Physiological responses of women to exercise. In: Wilmore JH, editor. Exercise and sports sciences reviews. Vol. 1. New York: Academic Press, 1973:126-54.

39. Franklin B, Buskirk E, Hodgson J, Gahagan H, Kollias J, Mendez J. Effects of physical conditioning on cardiorespiratory function, body composition and serum lipids in relatively normal weight and obese middle-age women. Int J Obes 1979;3:97-109.

40. Fringer MN, Stull AG. Changes in cardiorespiratory parameters during periods of training and detraining in young female adults. Med Sci Sports 1974;6:20-5.

41. Getchell LH, Moore JC. Physical training: comparative responses of middle-aged adults. Arch Phys Med Rehabil 1975;56:250-4.

42. Graves JE, Pollock ML, Leggett SH, Braith RW, Carpenter DM, Bishop LE. Effect of reduced training frequency on muscular strength. Int J Sports Med 1988;9:316-9.

43. Hagberg JM, Graves JE, Limacher M, et al. Cardiovascular responses of 70-79 year old men and women to exercise training. J Appl Physiol 1989;66:2589-94.

44. Hanson JS, Nedde WH. Long-term physical training effect in sedentary females. J Appl Physiol 1974;37:112-6.

45. Kearney JT, Stull AG, Ewing JL, Strein JW. Cardiorespiratory responses of sedentary college women as a function of training intensity. J Appl Physiol 1976;41:822-5.

46. Kilbom A. Physical training in women. Scand J Clin Lab Invest 1971; 119(Suppl):1-34.

47. Pels AE, Pollock ML, Dohmeier TE, Lemberger KA, Oehrlein BF. Effects of leg press training on cycling, leg press, and running peak cardiorespiratory measures. Med Sci Sports Exerc 1987;19:66-70.

48. Wilmore J. Inferiority of female athletes: myth or reality. J Sports Med 1974;3:1-6

49. American College of Sports Medicine. Guidelines for graded exercise testing and exercise prescription. $3^{\text {rd }}$ ed. Philadelphia: Lea \& Febiger, 1986.

50. Atomi Y, Ito K, Iwasaki H, Miyashita M. Effects of intensity and frequency of training an aerobic work capacity of young females. J Sports Med 1978;18:3-9.

51. Gettman LR, Pollock ML, Durstine JL, Ward A, Ayres J, Linnerud AC. Physiological responses of men to 1,3 , and 5 day per week training programs. Res Q 1976;47:638-46.

52. Hickson RC, Rosenkoetter MA. Reduced training frequencies and maintenance of increased aerobic power. Med Sci Sports Exerc 1981;13:136.

53. Hickson RC, Kanakis C, Davis JR, Moore AM, Rich S. Reduced training duration effects on aerobic power, endurance, and cardiac growth. J Appl Physiol 1982;53:225-9.

54. Hickson RC, Foster C, Pollock ML, Galassi TM, Rich S. Reduced training intensities and loss of aerobic power, endurance, and cardiac growth. J Appl Physiol 1985;58:492-9.

55. Pollock ML, Tiffany J, Gettman L, Janeway R, Lofland H. Effects of frequency of training on serum lipids, cardiovascular function, and body composition. In: Franks BD, editor. Exercise and fitness. Chicago: Athletic Institute, 1969:161-78.

56. Shephard RJ. Intensity, duration, and frequency of exercise as determinants of the response to a training regime. Int Z Angew Physiol 1969; 26:272-8.
57. Sidney KH, Eynon RB, Cunningham DA. Effect of frequency of training of exercise upon physical working performance and selected variables representative of cardiorespiratory fitness. In: Taylor AW, editor. Training scientific basis and application. Springfield, IL: Charles C. Thomas Co., 1972:144-88.

58. Crews TR, Roberts JA. Effects of interaction of frequency and intensity of training. Res Q 1976;47:48-55.

59. Davies CT, Knibbs AV. The training stimulus, the effects of intensity, duration and frequency of effort on maximum aerobic power output. Int Z Angew Physiol 1971;29:299-305.

60. Gossard W, Haskell WL, Taylor B, et al. Effects of low- and high-intensity home-based exercise training on functional capacity in healthy middle-age men. Am J Cardiol 1986;57:446-9.

61. Hollmann W, Venrath H. Die Beinflussung von Herzgrösse, maximaler $\mathrm{O}_{2}$ - Aufnahme und Ausdauergranze durch ein Ausdauertraining mittlerer und hoher Intensität. Der Sportarzt 1963;9:189-93.

62. Karvonen M, Kentala K, Mustala O. The effects of training heart rate: a longitudinal study. Ann Med Exp Biol Fenn 1957;35:307-15.

63. Kilbom A, Hartley L, Saltin B, Bjure J, Grimby G, Åstrand I. Physical training in sedentary middle-aged and older men. Scand J Clin Lab Invest 1969;24:315-22.

64. Olree HD, Corbin B, Penrod J, Smith C. Methods of achieving and maintaining physical fitness for prolonged space flight. Final Progress Rep. to NASA, Grant No. NGR-04-002-004, 1969.

65. Goode RC, Virgin A, Romet TT, et al. Effects of a short period of physical activity in adolescent boys and girls. Can J Appl Sports Sci 1976;1: 241-50.

66. Hartung GH, Smolensky MH, Harrist RB, Runge R. Effects of varied durations of training on improvement in cardiorespiratory endurance. $\mathrm{J}$ Hum Ergol 1977;6:61-8.

67. Liang MT, Alexander JF, Taylor HL, Serfrass RC, Leon AS, Stull GA. Aerobic training threshold, intensity duration, and frequency of exercise. Scand J Sports Sci 1982;4:5-8.

68. Terjung RL, Baldwin KM, Cooksey J, Samson B, Sutter RA. Cardiovascular adaptation to twelve minutes of mild daily exercise in middleaged sedentary men. J Am Geriatr Soc 1973;21:164-8.

69. Wilmore JH, Royce J, Girandola RN, Katch FI, Katch VL. Physiological alternatives resulting from a 10-week jogging program. Med Sci Sports 1970;2:7-14.

70. Barry AJ, Daly JW, Pruett EDR, et al. The effects of physical conditioning on older individuals. I. Work capacity, circulatory-respiratory function, and work electrocardiogram. J Gerontol 1966;21:182-91.

71. Davis JA, Frank MH, Whipp BJ, Wasserman K. Anaerobic threshold alterations caused by endurance training in middle-aged men. J Appl Physiol 1979;46:1039-49.

72. Gaesser GA, Rich RG. Effects of high- and low-intensity exercise training on aerobic capacity and blood lipids. Med Sci Sports Exerc 1984; 16:269-74

73. Golding L. Effects of physical training upon total serum cholesterol levels. Res Q 1961;32:499-505.

74. Hanson JS, Tabakin BS, Levy AM, Nedde W. Long-term physical training and cardiovascular dynamics in middle-aged men. Circulation 1968; 38:783-99.

75. Hartley LH, Grimby G, Kilbom A, et al. Physical training in sedentary middle-aged and older men. Scand J Clin Lab Invest 1969;24:335-44.

76. Huibregtse WH, Hartley HH, Jones LR, Doolittle WD, Criblez TL. Improvement of aerobic work capacity following non-strenuous exercise. Arch Environ Health 1973;27:12-5. 
77. Ismail AH, Corrigan D, McLeod DF. Effect of an eight-month exercise program on selected physiological, biochemical, and audiological variables in adult men. Br J Sports Med 1973;7:230-40.

78. Knehr CA, Dill DB, Neufeld W. Training and its effect on man at rest and at work. Am J Physiol 1942;136:148-56.

79. Leon AS, Conrad J, Hunninghake DB, Serfass R. Effects of a vigorous walking program on body composition, and carbohydrate and lipid metabolism of obese young men. Am J Clin Nutr 1979;32:1776-87.

80. Mann GV, Garrett LH, Farhi A, et al. Exercise to prevent coronary heart disease. Am J Med 1969;46:12-27.

81. Miyashita M, Haga S, Mitzuta T. Training and detraining effects on aerobic power in middle-aged and older men. J Sports Med 1978;18:1317.

82. Naughton J, Nagle F. Peak oxygen intake during physical fitness program for middle-aged men. JAMA 1965;191:899-901.

83. Oscai LB, Williams T, Hertig B. Effects of exercise on blood volume. J Appl Physiol 1968;24:622-4.

84. Pollock ML, Miller H, Janeway R, Linnerud AC, Robertson B, Valentino R.Effects of walking on body composition and cardiovascular function of middle-aged men. J Appl Physiol 1971;30:126-30.

85. Ribisl PM. Effects of training upon the maximal oxygen uptake of middle-aged men. Int Z Angew Physiol 1969;26:272-8.

86. Robinson S, Harmon PM. Lactic acid mechanism and certain properties of blood in relation to training. Am J Physiol 1941;132:757-69.

87. Santiago MC, Alexander JF, Stull GA, Serfrass RC, Hayday AM, Leon AS. Physiological responses of sedentary women to a 20 -week conditioning program of walking or jogging. Scand J Sports Sci 1987;9:33-9.

88. Shephard RJ. Future research on the quantifying of endurance training. J Hum Ergol 1975;3:163-81.

89. Skinner J, Holloszy J, Cureton T. Effects of a program of endurance exercise on physical work capacity and anthropometric measurements of fifteen middle-aged men. Am J Cardiol 1964:14:747-52.

90. Wood PD, Haskell WL, Blair SN, et al. Increased exercise level and plasma lipoprotein concentrations: a one-year, randomized, controlled study in sedentary, middle-aged men. Metabolism 1983;32:31-9.

91. Klissouras V, Pirnay F, Petit J. Adaptation to maximal effort: genetics and age. J Appl Physiol 1973;35:288-93.

92. Bouchard C. Gene-environment interaction in human adaptability. In: Malina RB, Eckert HM, editors. The academy papers. Champaign, IL: Human Kinetics Publishers, 1988:56-66.

93. Blair SN, Chandler JV, Ellisor DB, Langley J. Improving physical fitness by exercise training programs. South Med J 1980;73:1594-6.

94. Burke EJ, Franks BD. Changes in $\dot{V}_{2 \max }$ resulting from bicycle training at different intensities holding total mechanical work constant. Res Q 1975;46:31-7.

95. Pollock ML, Gettman LR, Raven PB, Ayres J, Bah M, Ward A. Physiological comparison of the effects of aerobic and anaerobic training. In: Price CS, Pollock ML, Gettman LR, Kent DA, editors. Physical fitness programs for law enforcement officers: a manual for police administrators. Washington, DC: U.S. Government Printing Office, No. 027-00000671-0, 1978:89-96.

96. Pollock ML, Ayres J, Ward A. Cardiorespiratory fitness: response to differing intensities and durations of training. Arch Phys Med Rehabil 1977; 58:467-73.

97. Siscovick DS, Weiss NS, Fletcher RH, Lasky T. The incidence of primary cardiac arrest during vigorous exercise. N Engl J Med 1984;311:8747.

98. Pollock ML. Prescribing exercise for fitness and adherence. In: Dishman RK, editor. Exercise adherence: its impact on public health. Champaign, IL: Human Kinetics Books, 1988:259-77.
99. Dishman RK, Sallis J, Orenstein D. The determinants of physical activity and exercise. Public Health Rep 1985;100:158-80.

100. Martin JE, Dubbert PM. Adherence to exercise. In: Terjung RL, editor. Exercise and sports sciences reviews. Vol. 13. New York: MacMillan Publishing Co., 1985:137-67.

101. Sallis JF, Haskell WL, Fortman SP, Vranizan KM, Taylor CB, Soloman DS. Predictors of adoption and maintenance of physical activity in a community sample. Prev Med 1986;15:131-41.

102. Devries HA. Exercise intensity threshold for improvement of cardiovascular-respiratory function in older men. Geriatrics 1971;26:94-101.

103. Wilmore JH, Ewy GA, Mortan AR, et al. The effect of beta-adrenergic blockade on submaximal and maximal exercise performance. J Cardiac Rehabil 1983;3:30-6.

104. Marigold EA. The effect of training at predetermined heart rate levels for sedentary college women. Med Sci Sports 1974;6:14-9.

105. Durnin JVGA, Passmore R. Energy, work and leisure. London, England: Heinemann Educational Books, Ltd., 1967:47-82.

106. Katch FI, McArdle WD. Nutrition, weight control and exercise. $3^{\text {rd }}$ ed. Philadelphia: Lea and Febiger, 1988:110-2.

107. Christensen EH. Physiological evaluation of work in the Nykroppa iron works. In: Floyd WF, Welford AT, editors. Ergonomics society symposium on fatigue. London, England: Lewis, 1953:93-108.

108. Pollock ML, Jackson AS, Foster C. The use of perception scale for exercise prescription. In: Borg G, Ottoson D, editors. The perception of exertion in physical work. London, England: MacMillan Press, Ltd., 1986:161-76

109. Borg GAV. Psychophysical bases of perceived exertion. Med Sci Sports Exerc 1982;14:377-81.

110. Borg G, Ottoson D, editors. The perception of exertion in physical work. London, England: The MacMillan Press, Ltd., 1986:4-7.

111. Birk TJ, Birk CA. Use of rating of perceived exertion for exercise prescription. Sports Med 1987;4:1-8.

112. Dishman RK, Patton RW, Smith J, Weinberg R, Jackson A. Using perceived exertion to prescribe and monitor exercise training heart rate. Int J Sports Med 1987;8:208-13

113. Chow JR, Wilmore JH. The regulation of exercise intensity by ratings of perceived exertion. J Cardiac Rehabil 1984;4:382-7.

114. Martin WH, Montgomery J, Snell PG, et al. Cardiovascular adaptations to intense swim training in sedentary middle-aged men and women. Circulation 1987:75:323-30.

115. Lieber DC, Lieber RL, Adams WC. Effects of run-training and swimtraining at similar absolute intensities on treadmill $\mathrm{V}_{2 \max }$. Med Sci Sports Exerc 1989;21:655-61.

116. Blair SN, Kohl HW, Goodyear NN. Rates and risks for running and exercise injuries: studies in three populations. Res Q Exerc Sports 1987; 58:221-8.

117. Oja P, Teraslinna P, Partanen T, Karava R. Feasibility of an 18 months' physical training program for middle-aged men and its effect on physical fitness. Am J Public Health 1975;64:459-65.

118. Pollock ML, Gettman R, Milesis CA, Bah MD, Durstine JL, Johnson RB. Effects of frequency and duration of training on attrition and incidence of injury. Med Sci Sports 1977;9:31-6.

119. Powell KE, Kohl HW, Caspersen CJ, Blair SN. An epidemiological perspective of the causes of running injuries. Phys Sports Med 1986; 14:100-14.

120. Richie DH, Kelso SF, Bellucci PA. Aerobic dance injuries: a retrospective study of instructors and participants. Phys Sports Med 1985;13: $130-40$ 
121. Delorme TL. Restoration of muscle power by heavy resistance exercise. J Bone Joint Surg 1945;27:645-67.

122. Gettman LR, Pollock ML. Circuit weight training: a critical review of its physiological benefits. Phys Sports Med 1981;9:44-60.

123. Mayhew JL, Gross PM. Body composition changes in young women with high resistance weight training. Res Q 1974;45:433-9.

124. Misner JE, Boileau RA, Massey BH, Mayhew JH. Alterations in body composition of adult men during selected physical training programs. J Am Geriatr Soc 1974;22:33-8.

125. Wilmore JH. Alterations in strength, body composition, and anthropometric measurements consequent to a 10 -week weight training program. Med Sci Sports 1974;6:133-8.

126. Allen TE, Byrd RJ, Smith DP. Hemodynamic consequences of circuit weight training. Res Q 1976;43:299-306.

127. Gettman LR, Ayres JJ, Pollock ML, Jackson A. The effect of circuit weight training on strength, cardiorespiratory function, and body composition of adult men. Med Sci Sports 1978;10:171-6.

128. Gettman LR, Ayres J, Pollock ML, Durstine JL, Grantham W. Physiological effects of circuit strength training and jogging. Arch Phys Med Rehabil 1979;60:115-20.

129. Gettman LR, Culter LA, Strathman T. Physiologic changes after 20 weeks of isotonic vs. isokinetic circuit training. J Sports Med Phys Fitness 1980;20:265-74.

130. Hurley BF, Seals DR, Ehsani AA, et al. Effects of high-intensity strength training on cardiovascular functions. Med Sci Sports Exerc 1984;16: 483-8.

131. Kimura Y, Itow H, Yamazakie S. The effects of circuit weight training on $\dot{\mathrm{VO}}_{2 \max }$ and body composition of trained and untrained college men. J Physiol Soc Jpn 1981;43:593-6.

132. Messier JP, Dill M. Alterations in strength and maximal oxygen uptake consequent to Nautilus circuit weight training. Res Q Exerc Sport 1985; 56:345-51.

133. Wilmore J, Parr RB, Vodak PA, et al. Strength, endurance, BMR, and body composition changes with circuit weight training. Med Sci Sports 1976;8:58-60.

134. Benestad AM. Trainability of old men. Acta Med Scand 1965;178:3217.

135. Devries HA. Physiological effects of an exercise training regimen upon men age 52 to 88 . J Gerontol 1970;24:325-36.

136. Hollmann W. Changes in the capacity for maximal and continuous effort in relation to age. In: Jok1 E, Simon E, editors. Int Res Sports Phys ed. Springfield, IL: Charles C. Thomas Co., 1964:369-71.

137. Skinner J. The cardiovascular system with aging and exercise. In: Brunner D, Jokl E, editors. Physical activity and aging. Baltimore: University Park Press, 1970:100-8.

138. Badenhop DT, Cleary PA, Schaal SF, Fox EL, Bartels RL. Physiology adjustments of higher- or lower-intensity exercise in elders. Med Sci Sports Exerc 1983;15:496-502.

139. Pollock ML, Dawson GA, Miller Jr HS, et al. Physiologic response of men 49 to 65 years of age to endurance training. J Am Geriatr Soc 1976;24:97-104.

140. Suominen H, Heikkinen E, Tarkatti T. Effect of eight weeks physical training on muscle and connective tissue of the $\mathrm{m}$. vastus lateralis in 69-year-old men and women. J Gerontol 1977;32:33-7.

141. Thomas SG, Cunningham DA, Rechnitzer PA, Donner AP, Howard JH. Determinants of the training response in elderly men. Med Sci Sports Exerc 1985;17:667-72.

142. Carter JE, Phillips WH. Structural changes in exercising middle-aged males during a 2-year period. J Appl Physiol 1969;27:787-94.
143. Kasch FW, Wallace JP. Physiological variables during 10 years of endurance exercise. Med Sci Sports 1976;8:5-8.

144. Kasch FW, Wallace JP, Van Camp SP. Effects of 18 years of endurance exercise on physical work capacity of older men. J Cardiopulmonary Rehabil 1985;5:308-12.

145. Dehn MM, Bruce RA. Longitudinal variations in maximal oxygen intake with age and activity. J Appl Physiol 1972;33:805-7.

146. Heath GW, Hagberg JM, Ehsani AA, Holloszy JO. A physiological comparison of young and older endurance athletes. J Appl Physiol 1981; 51:634-40.

147. Buskirk ER, Hodgson JL. Age and aerobic power: the rate of change in men and women. Fed Proc 1987;46:1824-9.

148. Drinkwater BL, Horvath SM, Wells CL. Aerobic power of females, ages 10 to 68. J Gerontol 1975;30:385-94.

149. Åstrand PO. Exercise physiology of the mature athlete. In: Sutton JR, Brock RM, editors. Sports medicine for the mature athletes. Indianapolis, IN: Benchmark Press, Inc., 1986:3-16.

150. Pollock ML, Foster C, Knapp D, Rod JS, Schmidt DH. Effect of age and training on aerobic capacity and body composition of master athletes. J Appl Physiol 1987;62:725-31.

151. Pollock ML, Miller Jr HS, Wilmore J. Physiological characteristics of champion American track athletes 40 to 70 years of age. J Gerontol 1974;29:645-9.

152. Brynteson P, Sinning WE. The effects of training frequencies on the retention of cardiovascular fitness. Med Sci Sports 1973;5:29-33.

153. Coyle EF, Martin WH, Sinacore DR, Joyner MJ, Hagberg JM, Holloszy JO. Time course of loss of adaptation after stopping prolonged intense endurance training. J Appl Physiol 1984;57:1857-64.

154. Cureton TK, Phillips EE. Physical fitness changes in middle-aged men attributable to equal eight-week periods of training, non-training and retraining. J Sports Med Phys Fitness 1964;4:1-7.

155. Knuttgen HG, Nordesjo LO, Ollander B, Saltin B. Physical conditioning through interval training with young male adults. Med Sci Sports 1973;5:220-6.

156. Roskamm H. Optimum patterns of exercise for healthy adults. Can Med Assoc 1967;96:895-9.

157. Saltin B, Blomqvist G, Mitchell J, Johnson RL, Wildenthal K, Chapman CB. Response to exercise after bed rest and after training. Circulation 1968;37,38(Suppl 7):1-78.

158. Kendrick ZB, Pollock ML, Hickman TN, Miller HS. Effects of training and detraining on cardiovascular efficiency. Am Corr Ther J 1971; 25:79-83.

159. Wilmore JH. Body composition in sport and exercise: directions for future research. Med Sci Sports Exerc 1983;15:21-31.

160. Wilmore JH, Royce J, Girandola RN, Katch FI, Katch VL. Body composition changes with a 10-week jogging program. Med Sci Sports 1970;2:113-7.

161. O'Hara W, Allen C, Shephard RJ. Loss of body weight and fat during exercise in a cold chamber. Eur J Appl Physiol 1977;37:205-18.

162. Zuti WB, Golding LA. Comparing diet and exercise as weight reduction tools. Phys Sports Med 1976;4:49-53.

163. American College of Sports Medicine. Proper and improper weight loss programs. Med Sci Sports Exerc 1983;15:9-13.

164. Hill JO, Sparling PB, Shields TW, Heller PA. Effects of exercise and food restriction on body composition and metabolic rate in obese women. Am J Clin Nutr 1987;46:622-30.

165. Pavlou KN, Steffee WP, Learman RH, Burrows BA. Effects of dieting and exercise on lean body mass, oxygen uptake, and strength. Med Sci Sports Exerc 1985;17:466-71. 
166. Gwinup G. Effect of exercise alone on the weight of obese women Arch Int Med 1975;135:676-80.

167. Sidney KH, Shephard RJ, Harrison J. Endurance training and body composition of the elderly. Am J Clin Nutr 1977;30:326-33.

168. Flint MM, Drinkwater BL, Horvath SM. Effects of training on women's response to submaximal exercise. Med Sci Sports 1974;6:89-94.

169. Girandola RN. Body composition changes in women: effects of high and low exercise intensity. Arch Phys Med Rehabil 1976;57:297-300.

170. Smith DP, Stransky FW. The effect of training and detraining on the body composition and cardiovascular response of young women to exercise. J Sports Med 1976;16:112-20.

171. Graves JE, Pollock ML, Jones AE, Colvin AB, Leggett SH. Specificity of limited range of motion variable resistance training. Med Sci Sports Exerc 1989;21:84-9.

172. Knapik JJ, Maudsley RH, Rammos NV. Angular specificity and test mode specificity of isometric and isokinetic strength training. J Orthop Sports Phys Ther 1983;5:58-65.

173. Edstrom L, Grimby L. Effect of exercise on the motor unit. Muscle Nerve 1986;9:104-26.

174. Hettinger T. Physiology of strength. Springfield, IL: C.C. Thomas Publishers, 1961:18-40.

175. Berger RA. Effect of varied weight training programs on strength. Res Q 1962;33:168-81.
176. MacDougall JD, Ward GR, Sale DG, Sutton JR. Biochemical adaptation of human skeletal muscle to heavy resistance training and immobilization. J Appl Physiol 1977;43:700-3.

177. Marcinik EJ, Hodgdon JA, Mittleman U, O'Brien JJ. Aerobic/calisthenic and aerobic/circuit weight training programs for Navy men: a comparative study. Med Sci Sports Exerc 1985;17:482-7.

178. Armstrong RB. Mechanisms of exercise-induced delayed onset muscular soreness: a brief review. Med Sci Sports Exerc 1984;16:529-38.

179. Jones DA, Newman DJ, Round JM, Tolfree SEL. Experimental human muscle damage: morphological changes in relation to other indices of damage. J Physiol (Lond) 1986;375:435-8.

180. Lewis SF, Taylor WF, Graham RM, Pettinger WA, Shutte JE, Blomqvist CG. Cardiovascular responses to exercise as functions of absolute and relative work load. J Appl Physiol 1983;54:1314-23.

181. MacDougall JD, Tuxen D, Sale DG, Moroz JR, Sutton JR. Arterial blood pressure response to heavy resistance training. J Appl Physiol 1985; 58:785-90

182. Hakkinen K. Factors influencing trainability of muscular strength during short term and prolonged training. Natl Strength Cond Assoc J $1985 ; 7: 32-4$

183. Gillam GM. Effects of frequency of weight training on muscle strangth enhancement. J Sports Med 1981;21:432-6.

184. Gettman LR, Ward P, Hagman RD. A comparison of combined running and weight training with circuit weight training. Med Sci Sports Exerc 1982;14:229-34. 